\title{
Entomofauna asociada al cultivo de achira Canna indica (Cannaceae) en tres zonas de Colombia
}

\author{
Associated entomofauna with edible canna crops Canna indica (Cannaceae) \\ in three areas of Colombia \\ MARÍA CAMILA ORTEGA ${ }^{1}$; SINDY LORENA MOJICA-RAMOS ${ }^{2}$; ERIKA VALENTINA \\ VERGARA-NAVARRO'; ${ }^{3}$ PAOLA SOTELO-CARDONA ${ }^{4}$
}

\begin{abstract}
${ }^{1}$ Profesional de Apoyo a la investigación. Corporación colombiana de investigación agropecuaria (Agrosavia), Centro de Investigación Obonuco, km 5 vía Pasto-Obonuco, Pasto, Nariño, Colombia, mcortega@agrosavia.co, https://orcid.org/0000-0001-8271-3175. ${ }^{2}$ Profesional de Apoyo a la investigación. Corporación colombiana de investigación agropecuaria (Agrosavia), Centro de Investigación Tibaitatá, $\mathrm{km} 14$ vía Mosquera-Bogotá, Mosquera, Cundinamarca Colombia, smojica@agrosavia.co, https://orcid.org/0000-0002-2493-6036. ${ }^{3}$ Investigador Master. Corporación colombiana de investigación agropecuaria (Agrosavia), Centro de Investigación Tibaitatá, Colección Taxonómica Nacional de Insectos "Luis María Murillo" (CTNI), km 14 vía Mosquera-Bogotá, Mosquera, Cundinamarca, Colombia, evvergara@agrosavia.co. https://orcid.org/0000-0002-8447-6844. ${ }^{4}$ Scientist-Entomology. The World Vegetable Center. Shanhua, Tainan, Taiwan 74151, paola.sotelo@worldveg.org, https://orcid.org/0000-0001-8159-1794.
\end{abstract}

\begin{abstract}
Autor para correspondencia: Erika Valentina Vergara-Navarro, Investigador Master. Corporación colombiana de investigación agropecuaria (Agrosavia), Centro de Investigación Tibaitatá, Colección Taxonómica Nacional de Insectos "Luis María Murillo" (CTNI), km 14 vía Mosquera-Bogotá, Mosquera, Cundinamarca, Colombia,evvergara@agrosavia.co. https:// orcid.org/0000-0002-8447-6844.
\end{abstract}

Citación sugerida / Suggested citation

ORTEGA, M. C.; MOJICA-RAMOS, S. L.; VERGARA-NAVARRO, E. V.; SOTELOCARDONA, P. 2020. Entomofauna asociada al cultivo de achira Canna indica (Cannaceae) en tres zonas de Colombia. Revista Colombiana de Entomología 46 (1): e10167. https://doi. org/10.25100/socolen.v46i1.10167

Recibido: 2-mar-2018

Aceptado: 11-sep-2019

Publicado: 24-jul-2020

Revista Colombiana de Entomología

ISSN (Impreso): 0120-0488

ISSN (En línea): 2665-4385

https://revistacolombianaentomologia.univalle.edu.co/

Open access

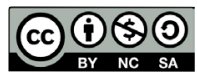

BY-NC-SA 4.0

Publicadores / Publishers:

Sociedad Colombiana de Entomología

SOCOLEN (Bogotá, D. C., Colombia)

http://www.socolen.org.co

Universidad del Valle (Cali, Colombia)

http://www.univalle.edu.co/

(C) 2020 Sociedad Colombiana de Entomología - SOCOLEN y Universidad del Valle - Univalle
Resumen: La achira o sagú (Canna indica) es una planta originaria de la cordillera de los Andes y cultivada en Colombia para la obtención de almidón a partir de sus rizomas. El almidón es utilizado en su mayoría como materia prima en la fabricación de bizcochos y otros productos autóctonos de consumo local y regional. Sin embargo, los productores de achira han mostrado reciente preocupación por la aparición de plagas que pueden ser limitantes en la productividad. El objetivo de este estudio consistió en reconocer los principales insectos asociados al cultivo. Se visitaron zonas productoras en los departamentos de Cundinamarca, Huila y Nariño. Se identificaron varios insectos nocivos del orden Lepidoptera en su estado larval, como Glyphipterix sp. (Glyphipterigidae), Spodoptera sp. (Noctuidae), Calpodes ethlius y Cobalus cannae (Hesperiidae), y el chinche Ischnodemus sp. (Hemiptera: Blissidae). También, entre otros insectos benéficos, se observó la emergencia de adultos de una avispa parasitoide de la familia Ichneumonidae a partir de larvas del microlepidóptero Glyphipterix sp., y se identificaron dos enemigos naturales de la chinche Ischnodemus sp., una avispa parasitoide de huevos (Hymenoptera: Chalcidoidea) y el hongo entomopatógeno Beauveria bassiana. Esta información puede servir como base de próximos estudios sobre aspectos de la biología y hábitos de los principales insectos nocivos de edible canna (Canna indica), así como de potenciales agentes de control biológico con el fin de generar recomendaciones de manejo en el cultivo de la achira.

Palabras clave: Glyphipterigidae, Blissidae, insectos nocivos, insectos benéficos, rizoma, raíces andinas.

Abstract: Edible canna (Canna indica) is a native crop from the Andes Cordillera and cultivated in Colombia to obtain starch from its rhizomes. Starch is used mostly as a raw material in biscuits and other local products for local and regional consumption manufacture. However, edible canna producers have shown recent concern about new pest effects that may be limiting crop productivity. This study aimed to identify the main insects associated with edible canna plants. Visits were made to production areas in departments of Cundinamarca, Huila, and Nariño. Several harmful Lepidoptera in their larval stage were identified, such as Glyphipterix sp. (Glyphipterigidae), Spodoptera sp. (Noctuidae), Calpodes ethlius and Cobalus cannae (Hesperiidae), and Ischnodemus sp. (Hemiptera: Blissidae). The emergence of adults of a parasitoid wasp of the family Ichneumonidae was observed from larvae of the microlepidopteran Glyphipterix sp. Two natural enemies of Ischnodemus sp., an egg-parasitoid wasp (Chalcidoidea), and the entomopathogenic fungus Beauveria bassiana were collected. Besides, other beneficial insects were collected. This information can serve as a basis for upcoming studies on the biology, and habits of the main insect pests of edible canna (Canna indica), as well as potential biological control agents to generate recommendations for control and management in edible canna crops.

Keywords: Glyphipterigidae, Blissidae, harmful insects, beneficial insects, rhizome, Andean roots.

\section{Introducción}

La achira o sagú (Canna indica L.) (Cannaceae, Zingiberales) es una de las raíces y tubérculos andinos nativos de importancia económica y nutricional para los agricultores de Los Andes (CIP 2015). Esta especie se cultiva en los valles 
interandinos templados desde México hasta el norte de Argentina (Fries 2007). La achira es una planta herbácea de reproducción vegetativa de cuyos rizomas cosechados entre los 10 y 14 meses después de siembra se obtiene almidón. Este es extraído y utilizado, principalmente, como materia prima en la fabricación de bizcochos y de otros productos autóctonos de consumo local y regional como bizcochuelos, pan, coladas y natillas. El rendimiento de rizoma del cultivo varía entre 15 y $40 \mathrm{t} /$ ha de materia fresca y un contenido de 10 a $17 \%$ de almidón (Fries 2007). En Colombia, es considerada una planta para cultivo de minifundio y huerta familiar, con extensiones promedio inferiores a una hectárea. Básicamente, es un cultivo de subsistencia en varios departamentos (Caicedo et al. 2003a) aunque también se cultiva con fines comerciales en Cundinamarca, Huila y, en menor medida, en Nariño, Cauca, Caquetá, Meta y Boyacá (Caicedo et al. 2003a; Rodríguez et al. 2003; Ministerio de Agricultura y Desarrollo Rural 2017).

La achira se adapta a una diversidad de climas y existen gran cantidad de morfotipos, tal y como se destaca en una investigación llevada a cabo en el Centro de Investigación La Selva (Rionegro, Antioquia), de la Corporación colombiana de investigación agropecuaria (Agrosavia). En dicha investigación se realizó la evaluación de la colección colombiana de achira, $C$. indica, con caracterización de variables como color de hoja, tallos, pétalos, sépalos, frutos, semilla, rizoma, forma de la hoja, fruto, rizoma y longitud, diámetro y número de tallos, rizoma, hojas, semilla, flores y fruto, encontrando una gran variabilidad fenotípica (LoboArias et al. 2017). El cultivo de la achira tiene una gran adaptabilidad climática y crece en varios pisos térmicos (0$2.650 \mathrm{msnm}$.), los cuales a su vez ofrecen una amplia gama de regímenes de lluvia, temperatura, humedad relativa y luminosidad en los que el cultivo presenta buen desarrollo. El cultivo de la achira está adaptado para crecer bajo regímenes de lluvia que van desde los 250 hasta $4.000 \mathrm{~mm}$ al año, temperaturas desde los $16{ }^{\circ} \mathrm{C}$ hasta $32{ }^{\circ} \mathrm{C}$, humedad relativa óptima entre $65 \%$ y $90 \%$ con una mínima del $40 \%$. Además, requiere gran luminosidad para realizar la fotosíntesis y obtener buena producción de almidón (Rodríguez et al. 2003). Debido a su rusticidad, no precisa mayores cuidados, salvo el apoyo con riego en períodos de sequía prolongada y la siembra en suelos con buena cantidad de materia orgánica (Fries 2007). Históricamente no se han tenido reportes sobre plagas que afecten seriamente la producción, aunque hay registro de algunos enrolladores, filófagos y de virosis en Colombia o en otros países andinos (Torres 1999; Torres 2004; Fries 2007). Sin embargo, Caicedo (2004) reportó la presencia ocasional de insectos como el gusano tornillo, Castniomera humboldtti (Boisduval, 1875) (Lepidoptera: Castniidae), chizas de las especies Platycoelia valida (Burmeister, 1844), Podischnus agenor (Olivier, 1789), Anomala sp. (Coleoptera: Scarabaeidae) y del gusano cogollero, Spodoptera sp. (Lepidoptera: Noctuidae). Desde 2014, diferentes actores de la cadena de achira expresaron su preocupación por la aparición de insectos nocivos que pueden ser limitantes en la productividad del cultivo y generaron demandas de investigación en este campo (Ministerio de Agricultura y Desarrollo Rural 2018). Los agricultores señalan que existen varios insectos plaga a nivel de follaje que deben monitorearse en el campo por su efecto dañino para el cultivo. Así, el reconocimiento de las potenciales plagas del cultivo de achira permitirá proponer prácticas de manejo y evitar pérdidas económicas en este cultivo promisorio, importante en la agroindustria rural de pequeña escala y de seguridad alimentaria de las regiones productoras. Por lo tanto, el objetivo de este trabajo fue identificar la entomofauna asociada al cultivo de achira en las principales áreas de producción en Colombia, y para tal fin se evaluaron fincas productoras en los departamentos de Cundinamarca, Huila y Nariño.

\section{Materiales y métodos}

Localización. La fase de campo se llevó a cabo en 24 fincas de los municipios de Fosca (4 $\left.{ }^{\circ} 18^{\prime} 17^{\prime \prime} \mathrm{N} 73^{\circ} 54^{\prime} 52^{\prime \prime} \mathrm{O}\right)$,

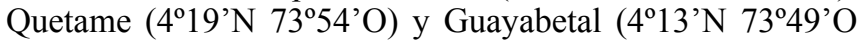
en Cundinamarca; cuatro fincas de las veredas Silvania y Obando en el municipio de Isnos (1 $1^{\circ} 58^{\prime} 02^{\prime} \mathrm{N} 76^{\circ} 15^{\prime} 2,8^{\prime \prime} \mathrm{O}$ ) y municipio de San Agustín ( $\left.1^{\circ} 52^{\prime} N 76^{\circ} 16^{\prime} O\right)$ en Huila; y en las veredas Buena Vista, Cofradía, Escandoy, Las Aradas, La Cañada, La Vega Alto, Llano Grande, Praditos, San Antonio, San Francisco y San Gerardo del municipio de La Cruz, Nariño (1 $\left.36^{\circ} \mathrm{N} 76^{\circ} 57^{\prime} \mathrm{O}\right)$, entre noviembre de 2015 y junio de 2016 (Fig. 1).

Toma de muestras. Los lotes visitados presentaban extensiones promedio inferiores a una hectárea. Estos se examinaron con el permiso de los agricultores y en su interior se realizaron recorridos en zig-zag, patrón comúnmente usado para búsqueda de insectos (Radcliffe et al. 2008). Se recolectaron plantas con daños asociados a la presencia de insectos en hojas, cogollos

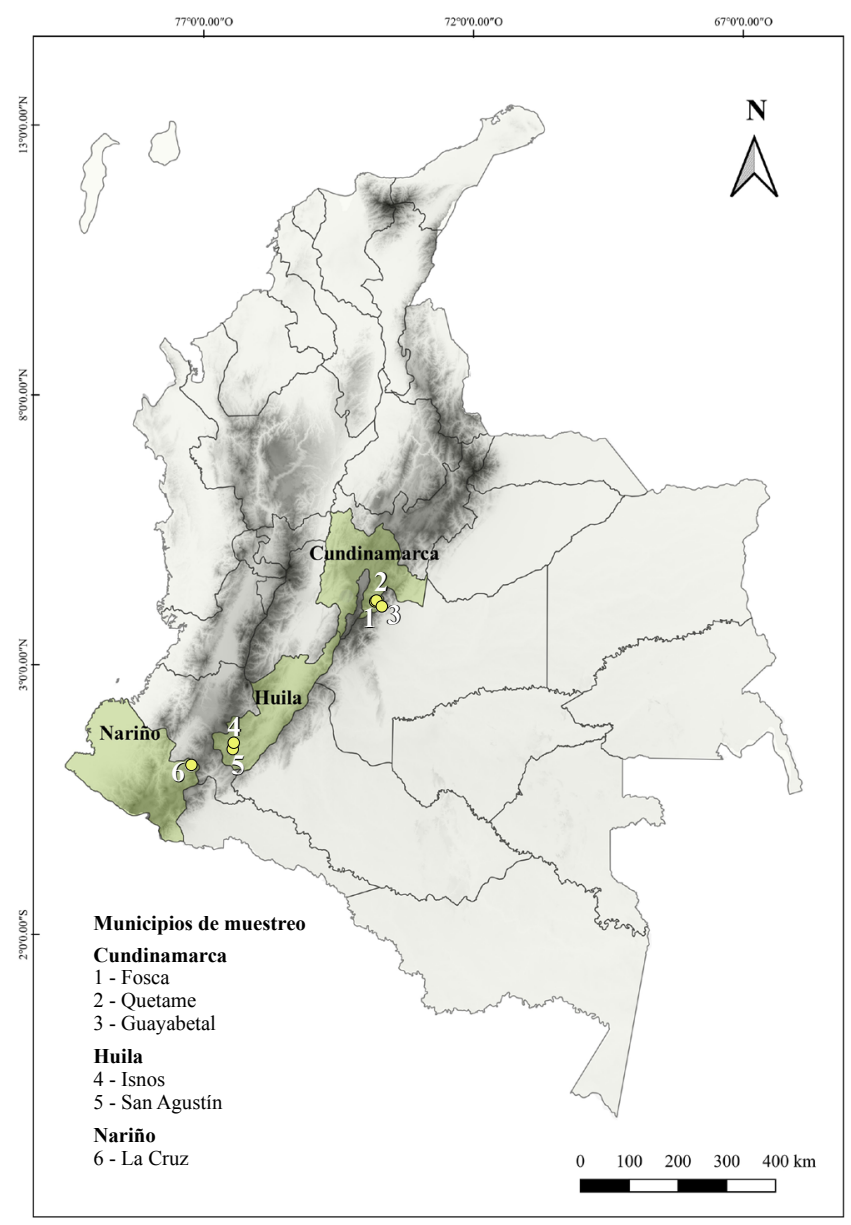

Figura 1. Ubicación de los puntos de recolección de especímenes en las tres regiones productoras de achira en Colombia. 

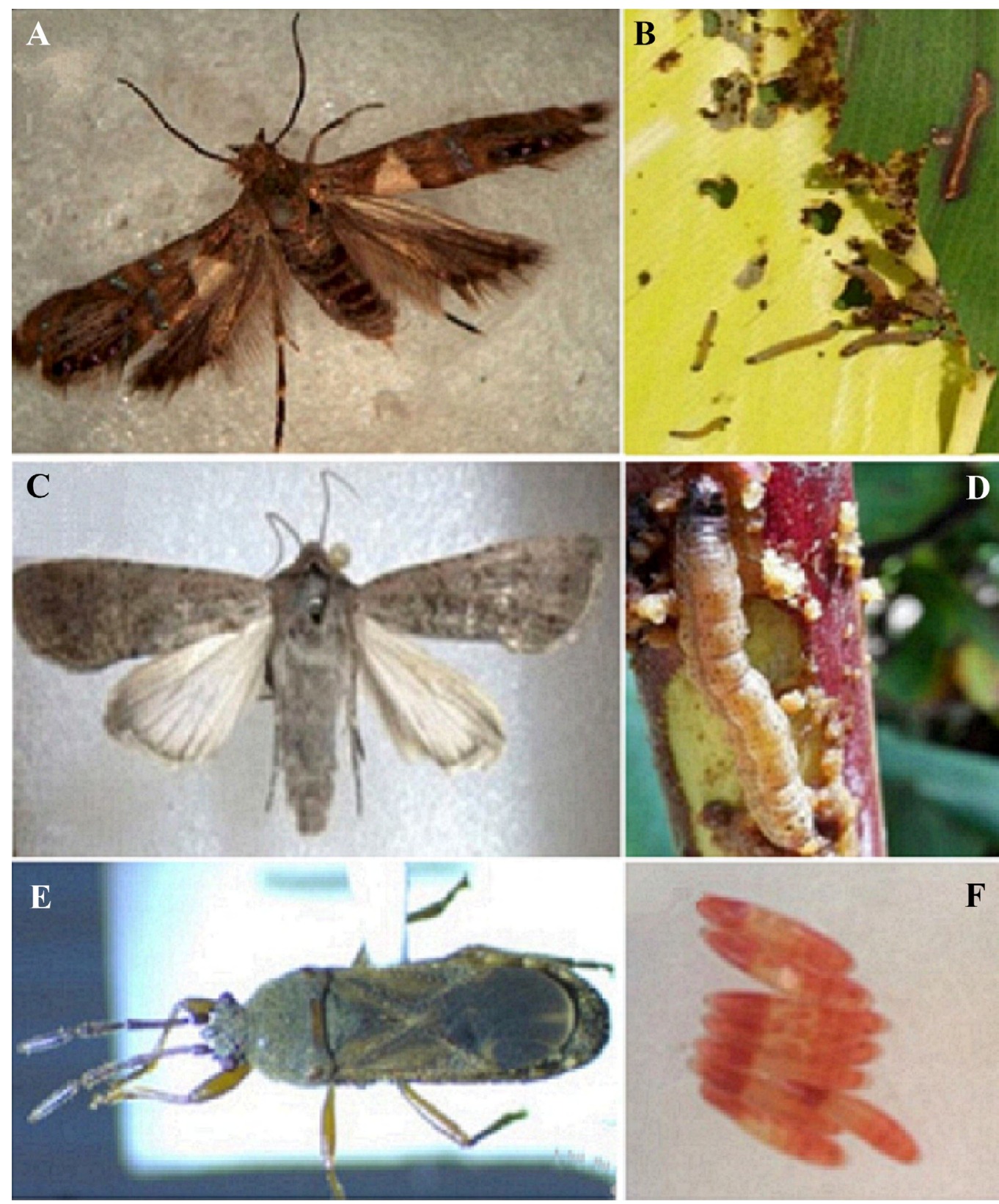

Figura 2. Insectos nocivos asociados al cultivo de achira. A. Adulto de Glyphipterix sp. B. Larvas de Glyphipterix sp. alimentándose de hojas jóvenes de achira. C. Adulto de Spodoptera sp. D. Larva de Spodoptera sp. alimentándose de tallos de achira. E. Adulto de Ischnodemus sp. F. Huevos de Ischnodemus sp.

y pseudotallos (Figs. 2B, 2C y 3). La recolección se realizó en plantas de achira en diferentes fases de crecimiento, a través de dos métodos de captura: barrido con jama y captura directa. El barrido con jama se hizo entre las 10:00-12:00 h y entre las 14:00-16:00 h, mediante 10 pases dobles durante un periodo aproximado de $30 \mathrm{~min}$ por lote. Posteriormente, los individuos recolectados se procesaron en una cámara letal con acetato de etilo y se trasladaron hasta el Laboratorio de Entomología del Centro de Investigación, Obonuco de Agrosavia (Nariño), para la fase preliminar de identificación. El segundo método usado para la recolecta de insectos fue captura directa sobre plantas y suelo, que consistió en buscar activamente los especímenes en el cultivo, sacudiendo las hojas sobre una superficie contrastante o en caso de encontrar daños en la planta asociados a presencia de insectos, removiendo la parte afectada de la planta, guardándola en frascos plásticos $\left(180 \mathrm{~cm}^{3}\right)$ diseñados para el transporte de insectos vivos y haciendo la respectiva rotulación de los recipientes. Los insectos recolectados en forma directa se transportaron vivos para ser criados como se explica a continuación.

Cría de micro y macrolepidópteros. Los insectos provenientes de campo se mantuvieron bajo condiciones controladas ofrecidas por una cámara climática (Modelo MLR-352H, Panasonic Biomedical). Las condiciones establecidas en la cámara correspondieron a las observadas en campo para temperatura, humedad relativa y fotoperiodo (Tabla 1). Los individuos recolectados se individualizaron en frascos de vidrio $\left(250 \mathrm{~cm}^{3}\right)$ cubiertos con muselina, rotulados con el lugar de procedencia y fecha de recolección. Los insectos se alimentaron semanalmente con hojas frescas de achira colectadas en el módulo de agricultura urbana del C. I. 
Tabla 1. Condiciones ofrecidas en la cámara climática para la cría de insectos plaga recolectados en campo.

\begin{tabular}{|c|c|c|c|c|c|c|}
\hline Hora & $6: 00$ & $8: 00$ & $10: 00$ & 12:00 & 18:00 & $5: 00$ \\
\hline Temperatura $\left({ }^{\circ} \mathrm{C}\right)$ & 18 & 19 & 22 & 25 & 18 & 18 \\
\hline Humedad (\% H. R.) & 60 & 50 & 50 & 50 & 60 & 60 \\
\hline $\operatorname{Luz}(\mathrm{LS})^{\mathrm{a}}$ & 2 & 3 & 3 & 3 & 0 & 1 \\
\hline
\end{tabular}

${ }^{a}$ Luz: Corresponde a bombillas fluorescentes de $40 \mathrm{~W}$, densidad fotosintética de flujo de fotones $150 \mu \mathrm{mol} \cdot \mathrm{m}^{-2} \cdot \mathrm{s}^{-1}$.

Obonuco de Agrosavia (Nariño). El material vegetal se lavó previamente con agua destilada para evitar contaminación de los insectos. Para cada individuo se hizo registro fotográfico, se obtuvieron medidas morfológicas (longitud y ancho) y se registró el tiempo para cambio de estados. Varios especímenes del microlepidóptero en estado adulto se enviaron a la Colección Taxonómica Nacional de Insectos "Luis María Murillo" (CTNI), C. I. Tibaitatá, Agrosavia para su posterior identificación y conservación.

Cría de chinches (Hemiptera). Los insectos se mantuvieron bajo las condiciones controladas descritas en la Tabla 1. Las chinches se ubicaron en cajas Petri con papel filtro humedecido con agua destilada, en grupos de tres adultos por caja $\left(1+, 2{ }^{\AA}\right)$ alimentados con cogollos de achira cada tres días. Con un estereoscopio (SMZ 168 - TP, Motic, China) se realizó el correspondiente registro fotográfico de adultos e individuos inmaduros. Varios especímenes de la chinche en estado adulto se enviaron a la CTNI para su posterior identificación y conservación.

Reconocimiento de enemigos naturales. En una primera fase, larvas de microlepidópteros recolectadas en campo se colocaron en recipientes de plástico con capacidad de 250 $\mathrm{cm}^{3}$, para identificar potenciales parasitoides. Estos últimos, fueron conservados en alcohol al $96 \%$ para su posterior identificación. Así mismo se hizo captura y conservación en alcohol (96 \%) de la entomofauna asociada al cultivo para determinar posibles enemigos naturales.

Identificación y curaduría de entomofauna asociada al cultivo de achira. Se estableció la familia de los especímenes recolectados y se realizó el registro fotográfico de los identificados a nivel de género. Confirmada la identificación, los especímenes fueron montados según los estándares internacionales de Museos Entomológicos y reposan en la CTNI. Para validar su identificación, los insectos plaga (Glyphipterigidae y Blissidae) fueron procesados y enviados al Museo Entomológico UNAB, de la Universidad Nacional de Colombia, sede Bogotá.

\section{Resultados y discusión}

Insectos potencialmente nocivos asociados al cultivo de achira. A continuación se describen los insectos asociados al cultivo que fueron encontrados causando daños en diferentes órganos de la planta, por lo que se describen como potencialmente nocivos; sin embargo, el establecimiento de sus incidencias y niveles de daño económico para ser calificados como plaga deberán considerarse en estudios futuros.

Dentro de las muestras de achira recolectadas en las tres principales zonas productoras de Colombia (Fig. 1), se encontró una especie de microlepidóptero y varias de macrolepidópteros. El primero se identificó como Glyphipterix sp. (Fig. 2A) (No. Catal. CTNI 2082) insecto cuya familia forma parte de la superfamilia Yponomeutoidea que se divide en dos: Orthoteliinae y Glyphipteriginae (Heppner 2008). Estos microlepidópteros son comúnmente conocidos como polillas de juncos debido a que se encuentran generalmente cerca o sobre varias especies estas ciperáceas (Heppner 2008). Los adultos de estas micropolillas presentan un tamaño aproximado de 7-9 mm, con una amplitud alar de 1,2-1,5 cm $(\mathrm{n}=10)$ de las larvas son de coloración café clara (longitud: $1,0 \pm 0,10 \mathrm{~cm}$; ancho $0,4 \pm 0,12 \mathrm{~cm} ; \mathrm{n}=24$ ) son reconocidas por su hábito barrenador en semillas, brotes terminales, tallos, ramas y hojas de los hospedantes (Fig. 3C). La mayoría de estos insectos depositan los huevos sobre las superficies de las plantas o en las nervaduras de las hojas, ya que el ovipositor típico de las hembras no está especializado para perforar (Heppner 1985).

En campo, se observó que las larvas de Glyphipterix perforan las hojas más jóvenes de la planta de achira, generando con ello pudrición del tejido foliar (Fig. 3B). Estas larvas por su comportamiento barrenador, descienden a través de las hojas más jóvenes que envuelven el pseudotallo para continuar con su perforación y próximas a empupar salen de allí para la construcción de una cámara pupal cercana a la pared del pseudotallo y la base de las hojas (P. Sotelo, obs. pers. 2016). Este comportamiento coincide con lo reportado por Heppner (1985) en otras especies de Glyphipterigidae que son barrenadoras de tallo. Las pupas presentan coloración marrón oscuro (longitud: $1,1 \pm 0,10 \mathrm{~cm}$; ancho $0,35 \pm 0,12 \mathrm{~cm}$; $\mathrm{n}=18$ ) y tarda aproximadamente 14 días (rango $=11-19$ días). No se obtuvieron posturas de los microlepidópteros adultos para estimar la duración total de los estados de desarrollo. La historia de vida de estas especies en zonas templadas en Europa y Norte América presenta una generación por año (Heppner 1985); sin embargo, observaciones sobre esta especie en achira sugieren un comportamiento multivoltino (P. Sotelo, obs. pers. 2016).

Hasta el momento, a nivel mundial los glypipterigidos no se han reconocido por ser plagas de importancia agrícola, salvo dos especies que afectan significativamente cultivos de pastos en Inglaterra y Nueva Zelanda (Heppner 1985). Además, algunos de los hospedantes reconocidos se encuentran dentro de los grupos de las familias Poaceae (Festuca sp., Luzula sp., Dactylis glomerata L., entre otras), Piperaceae (Piper aduncum L., P. auritum Kunth), Urticaceae (Urtica sp. y Boehmeria cylindrica (L.) Sw.) y Crassulaceae (Securigera sp., Sedum sp.) (Heppner 1985).

Por otro lado, entre las plagas que atacan el área foliar de la achira, se destacó el gusano cogollero, Spodoptera sp. (Fig. 2C), cuya presencia se vinculó principalmente al sistema de asocio de cultivos que caracteriza a los productores de achira, quienes, debido al prolongado ciclo de cultivo de la especie, con frecuencia realizan la siembra junto con otras de ciclo más corto como el maíz, lo cual coincide con los registros de Caicedo et al. (2003b). Las larvas de este género (Fig. 2D) se alimentan de 28 especies vegetales cultivadas, entre las cuales se destacan el maíz, sorgo, algodón, soya, higuerilla, tomate, caña de azúcar, arroz, maní, melón, girasol, entre otros. Cuando sus poblaciones logran altos niveles dentro de sus hospedantes y particularmente durante épocas de verano, este insecto puede moverse hacia otros cultivos (Posada 1989). También se registró la presencia de otras especies de macrolepidópteros, incluyendo a la larva conocida como 

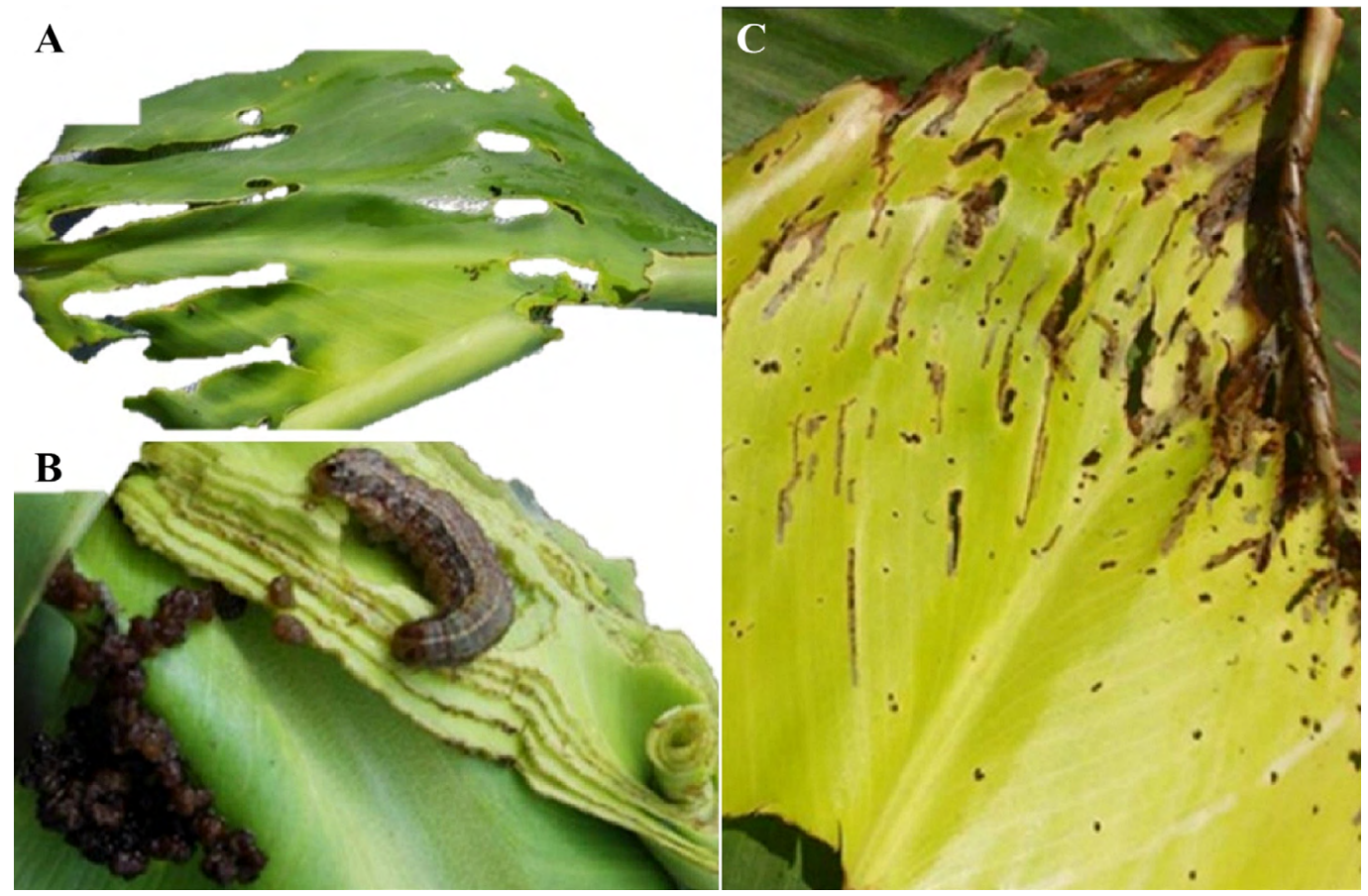

Figura 3. Daño causado por insectos nocivos de achira. A. y B. Daño característico de Spodoptera sp. C. Daño de Glyphipterix sp.

achirera, Calpodes ethlius (Stoll, 1782) y la larva de Cobalus cannae (Herrich-Schäffer, 1869), como lo registran varios autores (Montaldo 1991; Caicedo et al. 2003b; Caicedo 2004). Estos insectos se caracterizan por tener un vuelo muy rápido y errático, generalmente se alimentan de plantas dicotiledóneas. También se observaron dos especies de polillas de la familia Erebidae (Arctiinae), Anycles sp. y Dinia mena (Hübner, 1827). Las larvas de esta subfamilia se caracterizan por ser polífagas y pocas especies son de importancia económica.

En el departamento de Nariño se registró la presencia masiva de las chinches Blissidae Ischnodemus sp. (No. Catal. CTNI 2075, Fig. 2E). Las fincas visitadas mostraron incidencia variable de estos hemípteros, sin una preferencia marcada por alguna variedad. Los miembros de este género se caracterizan por presentar cuerpo alargado, delgado y aplanado lo cual se considera una ventaja para los insectos que viven dentro de los tallos de las plantas hospedantes. Así mismo, presentan antenas cilíndricas y cavidades coxales cerradas (Slater 1976). Como sucede típicamente con otros hemípteros, si las ninfas o los adultos son perturbados, secretan un fuerte olor de las glándulas olfativas localizadas en el tórax y el abdomen (Díaz et al. 2008; 2009). Los huevos (Fig. 2F) se encontraron en la base del pseudotallo en la zona axial, comportamiento que coincide con el lugar de oviposición reportado por otros autores para I. variegatus (Signator, 1857) (Díaz 2008; Montemayor et al. 2010; Brambila y Santana 2004).

Los miembros de la subfamilia Blissinae son un grupo de importancia económica (Schuh y Slater 1995). Ya que se hospedan y se alimentan de la savia de plantas monocotiledóneas. Se reportan en Gramineae, Cyperaceae, Restionaceae, Zingiberaceae, Juncaceae, Sparganiaceae, Typhaceae y Haemodoraceae. Sin embargo, la mayor cantidad de información sobre hospedantes se encuentra para varias especies de Poaceae (Slater 1976). En los estudios realizados por Overholt y colegas (2004), en Hymenachne amplexicauli, la presencia de la chinche $I$. variegatus disminuye la asimilación de dióxido de carbono, la tasa de crecimiento, la capacidad fotosintética y la biomasa. También, existen registros de la presencia de la especie I. fulvipes (De Geer) sobre hospedantes dentro de la familia de la achira, específicamente sobre $C$. indica (Slater y Wilcox 1966; Slater 1976). Las especies de Ischnodemus pueden presentar en algunos casos especificidad de alimentación (Slater y Wilcox 1966; Harrington 1972; Slater 1976) y podrían ser un potencial factor de disminución de rendimiento en diferentes cultivos.

Enemigos naturales de insectos nocivos asociados al cultivo de achira. En larvas del microlepidóptero Glyphipterix transportadas al laboratorio, donde alcanzaron su desarrollo hasta el estado de pupa se observó la emergencia de adultos de una avispa parasitoide de la familia Ichneumonidae (Figs. 4D, $4 \mathrm{E}$ y $4 \mathrm{~F}$ ) que podría pertenecer al género Pristomerus, el cual contiene alrededor de 100 especies a nivel mundial, la mayoría tropicales. Sus hospedantes son pequeños lepidópteros que viven en túneles, hojas enrolladas, brotes y otros refugios ( $\mathrm{Yu}$ et al. 2012).

Para el caso de la chinche Ischnodemus sp., se identificaron dos enemigos naturales: una avispa parasitoide de huevos (Hymenoptera: Chalcidoidea) (Figs. 4A y 4B) y el hongo entomopatógeno Beauveria bassiana (Deuteromycotina: Hyphomycetes) (Fig. 4C). Esta información coincide con la obtenida por Díaz y colegas (2009), quienes identificaron dos enemigos naturales para la especie I. variegatus en el Estado de la Florida (EE. UU.), el parasitoide de huevos Eumicrosoma sp. (Hymenoptera: Scelionidae) y el hongo entomopatógeno B. bassiana. Sin embargo, los estudios en campo por estos investigadores demostraron que el impacto de los enemigos naturales hacia las poblaciones de I. variegatus fue mínimo.

Otros insectos asociados al cultivo. Dentro de la entomofauna asociada a la achira se destaca la importancia de los insectos considerados benéficos. La reseña de los insectos 

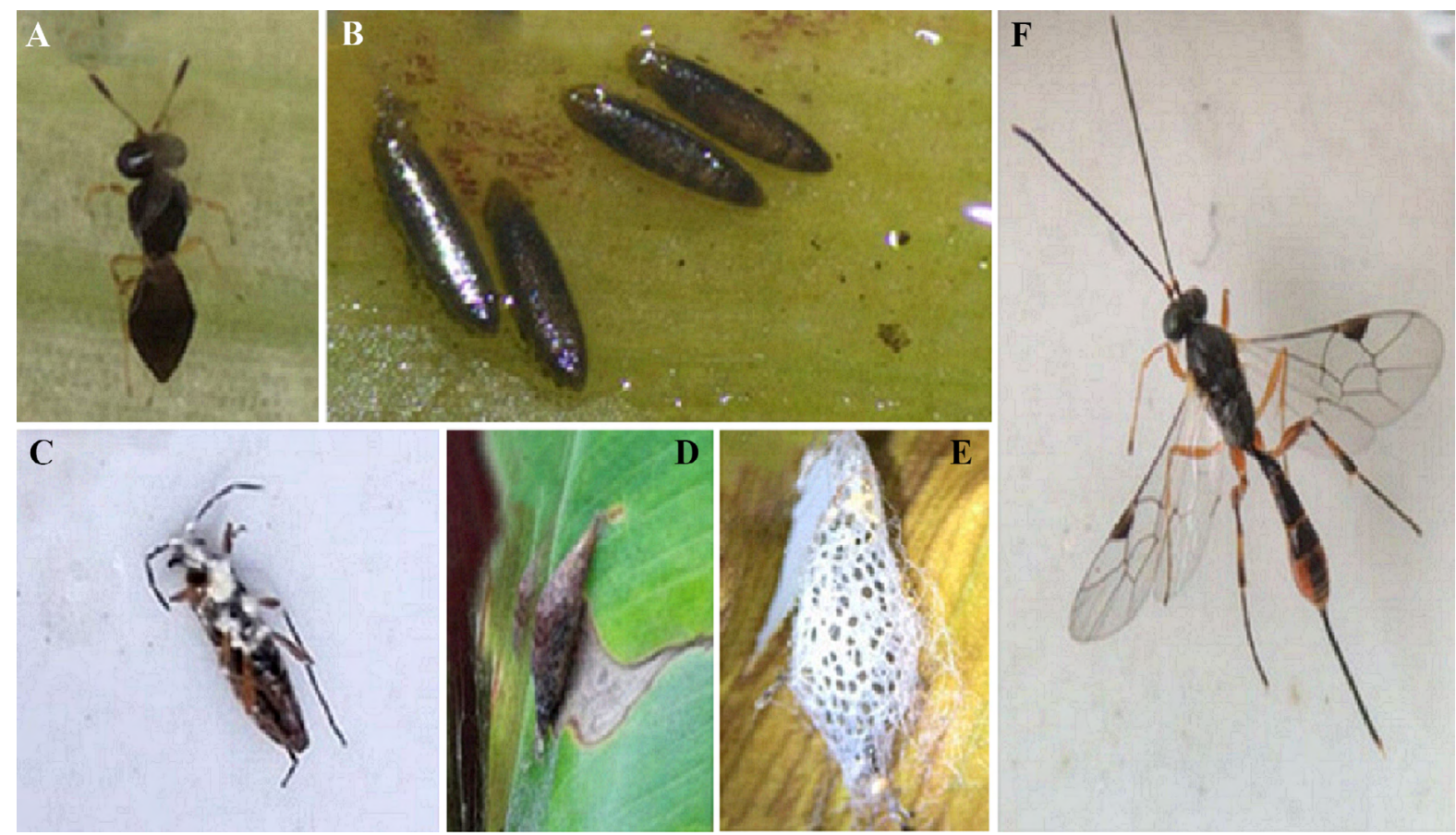

Figura 4. Enemigos naturales de insectos nocivos asociados al cultivo de achira. A. Avispa perteneciente a la familia Chalcidoidea, parasitoide de huevos de Ischnodemus sp. B. Huevos de Ischnodemus sp. parasitados por avispa Chalcidoidea. C. Muerte de Ischnodemus sp. por hongo Beauveria bassiana. D. Pupa de Glyphipterix sp. normal. E. Pupa de Glyphipterix sp. parasitada por Ichneumonidae. F. Adulto de Ichneumonidae posiblemente del género Pristomerus, parasitoide de Glyphipterix sp.

aquí mencionados proviene de DeBach y Rosen (1991), Flint y Dreistadt (1998) y Hajak (2004). Por órdenes taxonómicos, se encontraron:

Coleoptera: Hippodamia convergens (Guërin-Mëneville, 1824) (Coccinellidae) (No. Catal. CTNI 136, Fig. 5A), una de las especies más comúnmente encontradas en el continente americano, cuya función principal como controlador biológico de artrópodos considerados plaga en varios cultivos (áfidos, insectos escama, ácaros, etc.) es ampliamente conocida.

Se identificaron además individuos del género Diabrotica (Chrysomelidae) (No. Catal. CTNI 137, Fig. 5E) el cual incluye 338 especies que, frecuentemente, provocan daños subterráneos en cultivos comerciales.

Otro género de Chrysomelidae identificado fue Aspicela sp. (Chrysomelidae) (No. Catal. CTNI 144, Fig. 5F) del cual algunas especies han sido reportadas en bosques de niebla peruanos (Juárez y González 2017) y en malezas de clima medio del departamento de Nariño (Bravo 1970).

Hemiptera: Zelus longipes (Linnaeus, 1767) (Reduviidae) (No. Catal. CTNI 143, Fig. 5B), mejor conocidos como chinches asesinas. Dentro del género, varias especies son usadas como agentes de control biológico debido a su comportamiento depredador.

También se observaron individuos de Largus sp. (Largidae) (No. Catal. CTNI 146) género que incluye 61 especies, distribuidas desde el norte de EE. UU. hasta el centro de Argentina abarcando las Antillas (Stehlík y Kment 2010). Las especies de este género son de hábito fitófago y habitan generalmente el suelo, la hojarasca y plantas herbáceas, arbustos y árboles (Schuh y Slater 1995).

Se recolectaron individuos de dos especies de áfidos, uno reportado por Blackman y Eastop (2006) para C. indica: Macrosiphum euphorbiae (Thomas, 1878) (No. Catal. CTNI
2082) y una especie de Uroleucon sp. (No. Catal. CTNI 2083) sin registro previo para la familia Cannaceae.

Diptera: Se encontraron ejemplares de Palpada sp. (Syrphidae) (No. Catal. CTNI 145, Fig. 5D) que asemejan a las abejas o a las avispas. En estado larval son controladores biológicos, ya que depredan áfidos y otros insectos y como adultos se alimentan del néctar de flores.

Clausicella sp. (Tachinidae) (No. Catal. CTNI 2076, Fig. 5K) género de algunas especies que han desarrollado superparasitismos en plagas de importancia económica de cítricos como Ectomyelois ceratoniae (Lepidoptera: Phycitidae) y otras especies de interés (Kugler y Nitzan 1977).

Morellia sp. (Muscidae) (No. Catal. CTNI 2077, Fig. 5J) según Goto et al. (2012) este género es un eficiente polinizador de Thonningia sanguinea (Balanophoraceae), con la que ha coevolucionado en un sistema mutualista en el que la mosca oviposita y se alimenta de las inflorescencias masculinas en descomposición y no con anteras frescas, inflorescencias femeninas o frutos que son indispensables para la reproducción de la planta, por lo que el costo de la polinización es mínimo. También se ha reportado a Morellia como polinizador del género Heracleum sp. (Grace y Nelson 1981) (Apiaceaea).

Lonchaea sp. (Lonchaeidae) (No. Catal. CTNI 2078, Fig. 5I). Las especies de este género son de hábito saprofítico y pueden ser depredadoras de estados larvales de escarabajos xilófagos como Pissodes strobi (Peck, 1817) (Kovalev 1974; Alfaro y Borden 1980; Hulme 1990; Uchôa-Fernandes et al. 2003).

Dioxina sp. (Tephritidae) (No. Catal. CTNI 2079, Fig. 5L) del que se reportan algunas especies polinizadoras de orquídeas del género Schizochilus (Van der Niet et al. 2010).

Finalmente, se encontraron individuos de los géneros Eumecosomyia (No. Catal. CTNI 2080, Fig. 5G) y Chaetopsis (Diptera: Ulidiidae) (No. Catal. CTNI 2081, Fig. 5H), 

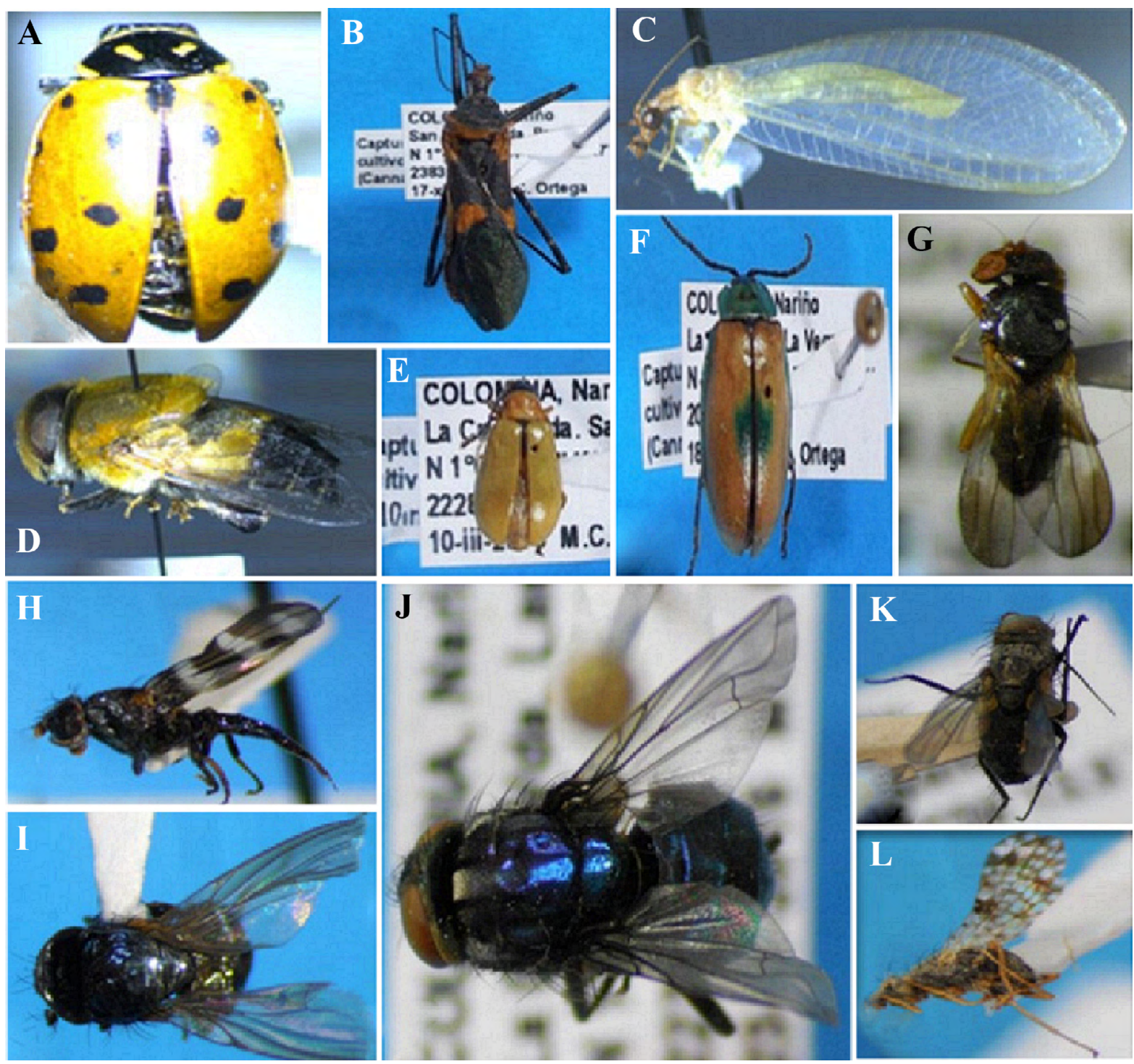

Figura 5. Otros insectos asociados al cultivo de la achira en tres zonas productoras de Colombia. A. Hippodamia convergens. B. Zelus longipes C. Chrysoperla sp. D. Palpada sp. E. Diabrotica sp. F. Aspicela sp. G. Eumecosomyia sp. H. Chaetopsis sp. I. Lonchaea sp. J. Morellia sp. K. Clausicela sp. L. Dioxina sp.|

reconocidas plagas de cultivos de maíz alrededor del mundo (Goyal et al. 2010; Cortez et al. 2013).

Neuroptera: Chrysoperla sp. (Chrysopidae) (No. Catal. CTNI 138, Fig. 5C), de cuya familia, las especies se encuentran distribuidas en forma cosmopolita y tanto larvas como adultos son depredadores de otros artrópodos de cuerpo blando.

\section{Conclusiones}

Se identificaron dos insectos potencialmente nocivos para el cultivo de la achira en las zonas productoras de Cundinamarca, Huila y Nariño: el microlepidóptero Glyphipterix sp. por el daño barrenador que ocasiona en los pseudotallos y la consecuente pudrición del cogollo, y la chinche Ischnodemus sp., chupadores importantes dada su abundancia. Para estas dos especies es necesario realizar estudios de su impacto en el rendimiento de almidón, de las pérdidas económicas en el cultivo por su incidencia y del impacto de las emergentes prácticas de manejo químico. De otra parte, dentro de la entomofauna asociada se registraron insectos benéficos pertenecientes a las familias Ichneumonidae y Chalcidoidea y el hongo entomopatógeno $B$. bassiana, los cuales pueden ser investigados a futuro como alternativa de control biológico para el manejo de insectos plaga en el cultivo de achira. Con nuevos estudios, se espera obtener mayor información sobre aspectos de la biología, hábitos de los principales insectos nocivos e insectos benéficos, con el fin de generar recomendaciones de manejo.

\section{Agradecimientos}

Los autores agradecen a Yuly Paola Sandoval por la toma de fotografías, al igual que a los taxónomos que contribuyeron en la identificación de algunas especies como fueron Javier Martínez-Alava y Juan Manuel Perilla (Diptera), Ronald Simbaqueba (Aphididae), Diana Isabel Rendón (Chrysopidae) y Guillermo González (Coccinellidae) y a los revisores anónimos por sus comentarios al manuscrito. 


\section{Literatura citada}

ALFARO, R. I.; BORDEN, J. H. 1980. Predation by Lonchaea corticis (Diptera: Lonchaeidae), on the white pine weevil, Pissodes strobi (Coleoptera, Curculionidae). The Canadian Entomologist 112 (12): 1259-1270. https://doi.org/10.4039/ Ent1121259-12

BLACKMAN, R. L.; EASTOP, V. F. 2006. Aphids on the world's Herbaceous plants and shrubs. Volume 1 - Host lists and keys. Volume 2 - The aphids. John Wiley \& Sons Ltd, Chichester and The Natural History Museum, London, England. 1456 p.

BRAMBILA, J.; SANTANA, F. J. 2004. Nota científica: First records for Ischnodemus variegatus (Hemiptera: Blissidae) in North America. The Florida Entomologist 87: 585-586. https:// doi.org/10.1653/0015-4040(2004)087[0585:FRFIVH]2.0.CO;2

BRAVO, G. 1970. Observaciones sobre el Dysonicha glabrata F. (Coleoptera: Chrysomelidae) como destructor de Amaranthus sp., una maleza común en la zona de clima medio de Nariño. Agricultura Tropical 26 (5): 227-231.

CAICEDO, G. E.; ROZO, L. S.; RENGIFO, G. 2003a. La achira. Técnicas de cultivo, beneficio o proceso pos cosecha y uso agroindustrial. Resultados de la investigación cofinanciada por el Programa Nacional de Transferencia de Tecnología Agropecuaria, Pronatta, Corpoica. Colombia: Huila, 86 p.

CAICEDO, G. E.; ROZO, L. S.; RENGIFO, G. 2003b. La achira: alternativa agroindustrial para áreas de economía campesina. Pronatta, Corpoica. Colombia, $87 \mathrm{p}$.

CAICEDO, G. E. 2004. El cultivo de achira: Alternativa de producción para el pequeño productor (11). pp. 149-156. En: Seminario, J. (Ed.). Raíces Andinas: Contribuciones al conocimiento y a la capacitación. Serie: Conservación y uso de la biodiversidad de raíces y tubérculos andinos: Una década de investigación para el desarrollo (1993-2003) No. 6. Universidad Nacional de Cajamarca, Centro Internacional de la Papa, Agencia Suiza para el Desarrollo y la Cooperación. Lima, Perú. $376 \mathrm{p}$.

CENTRO INTERNACIONAL DE LA PAPA (CIP). 2015. Raíces y tubérculos Andinos. Perú. Disponible en: https://cipotato.org/es/ raices-y-tuberculos-andinos/ [Fecha revisión: 10 enero 2018].

CORTEZ, E.; MEZA, J.; CAMACHO, J. 2013. La mosca de los estigmas [Chaetopsis massyla (Walker), Eumecosomyia nubila (Wiedemann) y Euxesta stigmatias (Loew)] en maíz; bioecología y manejo. pp. 153-169. En: Martínez, R.; Rojo, G. E.; García, C.; Ramírez, B.; Jasso, J.; Pérez, E.; González-Maldonado, M. B. Tecnologías de Granos y Semillas. Universidad Autónoma Indígena De México. 278 p.

DEBACH, P.; ROSEN, D. 1991. Biological control by natural enemies. Second edition. Cambridge University Press. New York, EE. UU., 386 p.

DÍAZ, R. 2008. Biology, host specificity and impact of Ischnodemus variegatus, a herbivore of Hymenachne amplexicaulis. $\mathrm{Ph} . \mathrm{D}$. thesis, University of Florida, Gainesville, FL., 187 p.

DÍAZ, R.; OVERHOLT, W. A.; CUDA, J. P.; PRATT, P. D.; FOX, A. 2008. Temperature-dependent development, survival, and potential distribution of Ischnodemus variegatus (Hemiptera: Blissidae), an herbivore of West Indian marsh grass. Annals of the Entomological Society of America 101 (3): 604-612. http://dx.doi. org/10.1603/0013-8746(2008)101[604:TDSAPD]2.0.CO;2

DÍAZ, R.; OVERHOLT, W. A.; CUDA, J. P.; PRATT, P. D.; FOX, A. 2009. Host specificity of Ischnodemus variegatus, an herbivore of West Indian marsh grass (Hymenachne amplexicaulis). BioControl 54: 307-321. https://doi.org/10.1007/s10526-0089188-3

FLINT, M. L.; DREISTADT, S. H. 1998. Natural enemies handbook: The illustrated guide to biological pest control. Oakland: Univ. Calif. Div. Agric. Nat. Res. Publ. 3386 p.

FRIES, A. M. 2007. Agronomía de los cultivos andinos. pp. 21-68. En: Tapia, M. E.; Fries, A. M. Guía de campo de los cultivos andinos. FAO y ANPE. Lima. 209 p.
GOTO, R.; YAMAKOSHI, G.; MATSUZAWA, T. 2012. A novel brood-site pollination mutualism?: the root holoparasite Thonningia sanguinea (Balanophoraceae) and an inflorescencefeeding fly in the tropical rainforests of West Africa. Plant Species Biology 27 (2): 164-169. https://doi.org/10.1111/j.14421984.2011.00338.x

GOYAL, G.; NUESSLY, G. S.; STECK, G. J.; SEAL, D. R.; CAPINERA, J. L.; BOOTE, K. J. 2010. New report of Chaetopsis massyla (Diptera: Ulidiidae) as a primary pest of corn in Florida. Florida Entomologist 93 (2): 198-202. https:// doi.org/10.1653/024.093.0208

GRACE, J.; NELSON, M. 1981. Insects and their pollen loads at a hybrid Heracleum site. New Phytologist 87 (2): 413-423. http:// dx.doi.org/10.1111/j.1469-8137.1981.tb03212.x

HAJAK, A. 2004. Natural enemies: An introduction to biological control. Cambridge University Press, New York. 378 p.

HARRINGTON, J. E. 1972. Notes on the biology of Ischnodemus species of America North of Mexico (Hemiptera: Lygaeidae: Blissinae). Occasional Papers Biological Science. University of Connecticut 2 (6): 47-56.

HEPPNER, J. B. 1985. The sedge moths of North America, Lepidoptera: Glyphipterigidae. Flora \& Fauna Handbook No. 1. Flora \& Fauna Publications, Gainesville, Florida, EE. UU. 257 p.

HEPPNER, J. B. 2008. Sedge moths (Lepidoptera: Glyphipterigidae). pp. 3335-3336. En: Capinera, J. L. (Ed.). Enclycopedia of Entomology. Second edition. Springer Science \& Bussiness Media. California, EE. UU. 4333 p.

HULME, M. A. 1990. Field assessment of predation by Lonchaea corticis (Diptera: Lonchaeidae) on Pissodes strobi (Coleoptera: Curculionidae) in Picea sitchensis. Environmental Entomology 19 (1): 54-58. https://doi.org/10.1093/ee/19.1.54

JUÁREZ, G.; GONZÁLEZ, U. 2017. Coleópteros (Insecta: Coleoptera) del bosque de neblina de Cuyas, Ayabaca-región Piura, Perú. The Biologist 14 (2): 199-217.

KOVALEV, V. G. 1974. Species of the genus Lonchaea Fallén close to L. limatula Collin (Diptera, Lonchaeidae). Revista de Entomología 53: 447-453.

KUGLER, J.; NITZAN, Y. 1977. Biology of Clausicella suturata (Diptera: Tachinidae) a parasite of Ectomyelois ceratoniae (Lepidoptera.: Phycitidae) BioControl 22 (1): 93-105.

LOBO-ARIAS, M.; MEDINA-CANO, C. I.; GRISALES-ARIAS, J. D.; YEPES-AGUDELO, A. F.; ÁLVAREZ-GUZMÁN, J. A. 2017. Caracterización y evaluación morfológicas de la colección colombiana de achira, Canna edulis Ker Gawl. (Cannaceae). Corpoica Ciencia y Tecnología Agropecuaria 18 (1): 47-73. http://dx.doi.org/10.21930/rcta.vol18_num1_ art:558

MINISTERIO DE AGRICULTURA Y DESARROLLO RURAL. 2017. Evaluaciones Agropecuarias Municipales. Disponible en: http://www.agronet.gov.co/estadistica/Paginas/default.aspx [Fecha revisión: 4 enero 2018].

MINISTERIO DE AGRICULTURA Y DESARROLLO RURAL. 2018. Plataforma Siembra. Disponible en: http://www.siembra. gov.co/Demandas/Demanda/Reporte [Fecha revisión: 5 diciembre 2018].

MONTALDO, A. 1991. Cultivo de raíces y tubérculos. Instituto Interamericano de Cooperación para la Agricultura. San José, Costa Rica. 408 p.

MONTEMAYOR, C. O.; DIAZ, R.; OVERHOLT, W. A.; HODGES, A.; CUDA, J. P. 2010. Myakka bug, Ischnodemus variegatus (Signoret) (Insecta: Hemiptera: Blissidae). IFAS Extension University of Florida. 1-6. Disponible en: http://edis.ifas.ufl.edu/ pdffiles/IN/IN84200.pdf [Fecha revisión: 12 marzo 2018].

OVERHOLT, W. A.; EWE, S. M. L.; DIAZ, R.; MORGAN, E. C.; MOERI, O. E. 2004. Feeding effects of Ischnodemus variegatus (Hemiptera: Blissidae) on photosynthesis and growth of Hymenachne amplexicaulis (Poaceae). The Florida Entomologist 87 (3): 312-316. https://doi.org/10.1653/00154040(2004)087[0312:FEOIVH]2.0.CO;2 
POSADA, L. 1989. Lista de insectos dañinos y otras plagas en Colombia. Instituto Colombiano Agropecuario, ICA. Boletín Técnico No. 43. Produmedios, Bogotá. 662 p.

RADCLIFFE, E. B.; HUTCHISON, W. D.; CANCELADO, R. E. (Eds.). 2008. Radcliffe's IPM World Textbook [En línea]. University of Minnesota, St. Paul, MN. Disponible en: http:// ipmworld.umn.edu/ [Fecha revisión: 21 diciembre 2016].

RODRÍGUEZ, G.; GARCÍA, H.; CAMACHO, J. H.; ARIAS, F. L. 2003. El almidón de achira o sagú (Canna edulis Ker): Manual técnico para su elaboración. Corporación Colombiana de Investigación Agropecuaria, Corpoica. Tibaitatá, Colombia, 33 p. Disponible en: http://bibliotecadigital.agronet.gov.co/ handle/11348/3744 [Fecha revisión: 17 agosto 2017].

SCHUH, R.; SLATER, J. 1995. True bugs of the world (Hemiptera Heteroptera). Cornell University press. Ithaca. $336 \mathrm{p}$.

SLATER, J. A. 1976. Monocots and chinch bugs: a study of host plant relationships in the Lygaeid subfamily Blissinae (Hempitera: Lygaeidae). Biotropica 8 (3): 143-165. https://doi. org/10.2307/2989681

SLATER, J. A.; WILCOX, D. B. 1966. An analysis of three new genera of Neotropical Blissinae (Hemiptera: Lygaeidae). Annals of the Entomological Society of America 50: 61-76.

STEHLÍK, J. L.; KMENT, P. 2010. Largus giganteus sp. nov. from Brazil and notes on hybridization within Largus (Hemiptera: Heteroptera: Largidae). Acta Entomologica Musei Nationalis Pragae 50: 53-58.

TORRES, L. J. 1999. Achira cultivo promisorio en Colombia. Corpoica, Regional Uno. Sena, Creced Oriente de Cundinamarca. Produmedios, Bogotá. 23 p.

TORRES, L. J. 2004. Tecnología para el cultivo de sagú o achira (Canna edulis Ker.). Corpoica y departamento de Cundinamarca. Secretaría de Agricultura y Desarrollo Rural, Bogotá. 39 p.

UCHÔA-FERNANDES, M. A.; DE OLIVEIRA, I.; MOLINA, R. M. S.; ZUCCHI, R. A. 2003. Biodiversity of frugivorous flies
(Diptera: Tephritoidea) captured in citrus groves, Mato Grosso do Sul, Brazil. Neotropical Entomology 32: 239-246. http:// dx.doi.org/10.1590/S1519-566X2003000200008

VAN DER NIET, T.; JÜRGENS, A.; JOHNSON, S. D. 2010. Pollinators, floral morphology and scent chemistry in the southern African orchid genus Schizochilus. South African Journal of Botany 76 (4): 726-738. https://doi.org/10.1016/j. sajb.2010.07.004

YU, D. S.; VAN ACHTERBERG, C.; HORSTMANN, K. 2012. Taxapad 2012. Ichneumonoidea 2011. Ottawa, Ontario, Canada Database on flash-drive. [Fecha revisión: 15 marzo 2018].

\section{Origen y financiación}

El trabajo fue realizado dentro del Macroproyecto "Tecnología para el fortalecimiento y modernización de la cadena agroindustrial de la achira o sagú (Canna edulis) en Colombia" financiado por Ministerio de Agricultura y Desarrollo Rural (MADR) con el soporte técnico de la Corporación colombiana de investigación agropecuaria (Agrosavia).

\section{Contribución de los autores}

María Camila Ortega: Muestreo, trabajo de laboratorio, escritura del artículo.

Sindy Lorena Mojica-Ramos: Muestreo, trabajo de laboratorio, escritura del artículo.

Erika Valentina Vergara-Navarro: Trabajo de laboratorio e identificación de especímenes, escritura del artículo.

Paola Sotelo-Cardona: Muestreo, trabajo de laboratorio, análisis de datos, escritura del artículo. 